\title{
ON ANOMALOUS LIEB-ROBINSON BOUNDS FOR THE FIBONACCI XY CHAIN
}

\author{
DAVID DAMANIK, MARIUS LEMM, MILIVOJE LUKIC, AND WILLIAM YESSEN
}

\begin{abstract}
We rigorously prove a new kind of anomalous (or sub-ballistic) Lieb-Robinson bound for the isotropic XY chain with Fibonacci external magnetic field at arbitrary coupling. It is anomalous in that the usual exponential decay in $x-v t$ is replaced by exponential decay in $x-v t^{\alpha}$ with $0<\alpha<1$. In fact, we can characterize the values of $\alpha$ for which such a bound holds as those exceeding $\alpha_{u}^{+}$, the upper transport exponent of the one-body Fibonacci Hamiltonian. Following the approach of [12], we relate Lieb-Robinson bounds to dynamical bounds for the one-body Hamiltonian corresponding to the XY chain via the Jordan-Wigner transformation; in our case the one-body Hamiltonian with Fibonacci potential. We can bound its dynamics by adapting techniques developed in $[6,7,2,3]$ to our purposes.

We also discuss the extension to the more general class of Sturmian potentials and we explain why our method does not extend to yield anomalous Lieb-Robinson bounds of power-law type for the random dimer model.
\end{abstract}

\section{Contents}

1. The Fibonacci XY Chain 2

2. Main Results 3

2.1. Anomalous Lieb-Robinson Bounds 3

2.2. Equality of Transport Exponents 5

3. The Relation to One-Body Dynamics 5

3.1. Diagonalizing the XY Chain 5

3.2. Relating LR Bounds for the XY Chain to Fermionic LR Bounds 6

4. Proof of the Second Main Result 7

5. Proof of the First Main Result 8

5.1. The Transport Exponent in a New Guise 8

5.2. Key Result: Fermionic LR Bounds from One-Body Dynamics 8

6. Proof of the Key Result 9

6.1. Strategy of Proof 9

6.2. The Dunford Functional Calculus Approach to Quantum Dynamics 9

6.3. Extension to the Half-Line Fibonacci Hamiltonian 11

6.4. Covariance of $H(\omega)$ under Shifts 12

6.5. Bounding Resolvent Matrix Elements by Transfer Matrix Norms 12

6.6. Lower Bounds on Transfer Matrix Norms and Conclusion 14

7. Proof that $\alpha^{\prime}=\alpha_{u}^{+} \quad 17$

8. The General Sturmian Case 18

2010 Mathematics Subject Classification. 47B36, 82B44.

D.Damanik was supported in part by NSF grant DMS-1067988, M.Lukic was supported in part by NSF grant DMS-1301582, and W.Yessen was supported by NSF grant DMS-1304287. 
9. Remark on the Random Dimer Model

References

\section{The Fibonacci XY Chain}

Given an integer $n$, we take as our Hilbert space

$$
\mathcal{H}_{n}=\bigotimes_{j=1}^{n} \mathfrak{h}_{j}
$$

where $\mathfrak{h}_{j}=\mathbb{C}^{2}$ for all $j$. On $\mathcal{H}_{n}$, we consider the isotropic XY chain given by the Hamiltonian

$$
H_{n}^{X Y}=-\sum_{j=1}^{n-1}\left(\sigma_{j}^{x} \sigma_{j+1}^{x}+\sigma_{j}^{y} \sigma_{j+1}^{y}\right)+\sum_{j=1}^{n} V_{j} \sigma_{j}^{z}
$$

where $\left\{V_{j}\right\}$ is the Fibonacci external magnetic field defined by

$$
V_{j}=\lambda \chi_{\left[1-\phi^{-1}, 1\right)}\left(j \phi^{-1}+\omega \bmod 1\right)
$$

with $\lambda>0$ a coupling constant, $\omega \in[0,1)$ the "phase" and

$$
\phi=\frac{1+\sqrt{5}}{2}
$$

the golden mean. The Fibonacci external field is the primary model of onedimensional quasi-periodicity. As usual, the Pauli matrices are

$$
\sigma^{x}=\left(\begin{array}{ll}
0 & 1 \\
1 & 0
\end{array}\right), \quad \sigma^{y}=\left(\begin{array}{cc}
0 & -i \\
i & 0
\end{array}\right), \quad \sigma^{z}=\left(\begin{array}{cc}
1 & 0 \\
0 & -1
\end{array}\right),
$$

and $\sigma_{j}^{x, y, z}$ denotes $\mathbb{1}_{1} \otimes \ldots \mathbb{1}_{j-1} \otimes \sigma^{x, y, z} \otimes \mathbb{1}_{j+1} \ldots \otimes \mathbb{1}_{n}$. For a finite set $S \subset \mathbb{Z}_{+}$, we define the algebra of observables on $J$ by

$$
\mathcal{A}_{J}=\bigotimes_{j \in J} \mathcal{B}\left(\mathfrak{h}_{j}\right)
$$

where $\mathcal{B}\left(\mathfrak{h}_{j}\right)$ is the set of bounded linear operators on $\mathfrak{h}_{j}=\mathbb{C}^{2}$, which is of course just the set of all complex $2 \times 2$ matrices. We will often make use of the fact that for $J \subset J^{\prime}$, there is a natural embedding of $\mathcal{A}_{J}$ into $\mathcal{A}_{J^{\prime}}$ by tensoring with the identity on $J^{\prime} \backslash J$. Also, we denote $\mathcal{A}_{j} \equiv \mathcal{A}_{\{j\}}$.

Finally, we recall that the Heisenberg dynamics of an observable $A \in \mathcal{A}_{J}$ are defined by

$$
\tau_{t}^{n}(A)=e^{i t H_{n}^{X Y}} A e^{-i t H_{n}^{X Y}}
$$

Disclaimer We usually do not keep track of constants that depend on model parameters, one exception is the dependence on the parameter $\omega$ as discussed later. We write $C, C^{\prime}, \ldots$ for constants that may have different numerical values from line to line and $C_{0}, C_{1}, \ldots$ for constants that appear in the statement of a result. 


\section{Main Results}

2.1. Anomalous Lieb-Robinson Bounds. Lieb-Robinson (LR) bounds, first introduced by Lieb and Robinson in 1972 [19], quantify the speed of propagation of the Heisenberg dynamics in quantum spin systems, up to exponential errors. Interest in LR bounds re-surged about 10 years ago, when Hastings [13] showed that they can be used to give exponential clustering for gapped quantum spin systems, see also [21]. We take a different perspective here and view LR bounds as a means of quantifying the dynamical properties of interacting many-body quantum systems.

To phrase our results, it will be convenient to adopt the following convention for stating anomalous LR bounds (we will soon discuss what we mean by "anomalous"):

Definition 2.1. We say that " $L R(\alpha)$ " holds if there exist non-negative constants $C_{0}, \mu, v$ such that for all integers $1 \leq j<j^{\prime} \leq n$ and all $t>0$, we have

$$
\left\|\left[\tau_{t}^{n}(A), B\right]\right\| \leq C_{0}\|A\|\|B\| e^{-\mu\left(\left|j^{\prime}-j\right|-v t^{\alpha}\right)}
$$

for all $A \in \mathcal{A}_{j}$ and all $B \in \mathcal{A}_{\left\{j^{\prime}, \ldots, n\right\}}$.

Remark 2.2. (i) The ordinary LR bound is $L R(1)$ and by the very general analysis of [21], $L R(1)$ holds in our case ${ }^{1}$. Of course, $L R(\alpha)$ with $\alpha<1$ is a stronger bound than $L R(1)$.

(ii) The assumption that $A \in \mathcal{A}_{j}$ can be generalized to $A \in \mathcal{A}_{J}$ with $\max J \leq j$ using the Leibniz rule for commutators (7), but at the price of having $C_{0}$ depend on $|J|$. For a detailed proof of this, we refer to [5]. In a different approach, following the proof of Corollary 3.4 in [12], one can derive from $L R(\alpha)$ a Lieb-Robinson bound which holds for all $A \in \mathcal{A}_{J}$ with $\max J \leq j$ and $C$ independent of $|J|$, but at the price of increasing the growth in $t$ to $\int_{0}^{t} e^{\mu v s^{\alpha}} \mathrm{d} s$.

We will phrase our results in terms of the upper transport exponent $\alpha_{u}^{+}$for the Fibonacci Hamiltonian $H$. It is just one of several exponents characterizing the dynamics associated to $H$ and we will introduce these later, in Section 7, mainly as tools.

Definition 2.3. Let $H$ be the operator on $\ell^{2}\left(\mathbb{Z}_{+}\right)$defined in (18) and let $\left\{\delta_{l}\right\}_{l \geq 1}$ be the canonical basis of $\ell^{2}\left(\mathbb{Z}_{+}\right)$. For $t>0$ and any integer $N$, we define

$$
P(N, t)=\sum_{n>N}\left|\left\langle\delta_{1}, e^{-i t H} \delta_{n}\right\rangle\right|^{2}, \quad S^{+}(\alpha)=-\limsup _{t \rightarrow \infty} \frac{\log P\left(t^{\alpha}-1, t\right)}{\log t}
$$

as well as the upper transport exponent

$$
\alpha_{u}^{+}=\sup _{\alpha \geq 0}\left\{S^{+}(\alpha)<\infty\right\}
$$

which, roughly speaking, characterizes the propagation rate of the fastest part of the wavepacket initally localized at $\delta_{1}$.

Theorem 2.4 (First main result). Let $\lambda>0$. If $\alpha>\alpha_{u}^{+}(\lambda)$, then $L R(\alpha)$.

Remark 2.5. The proof also yields the explicit formulae (38) for $\mu$ and for the "LiebRobinson velocity" $v$. The formula for $v$ does not yield quantitative information however, because it involves the quantity $C_{\delta}^{\prime}$, which is not determined in [3].

\footnotetext{
${ }^{1}$ In fact even the slightly stronger version of $L R(1)$ with $A \in \mathcal{A}_{J}$ and $C$ independent of the cardinality $|J|$ holds.
} 
Our second main result says that the upper transport exponent is truly the "correct" one for the LR bound (modulo the difference between $\geq$ and $>$ ).

Theorem 2.6 (Second main result). Let $\lambda>0$. If $L R(\alpha)$, then $\alpha \geq \alpha_{u}^{+}(\lambda)$.

Remark 2.7. The proof of Theorem 2.6 works in complete generality and has nothing to do with the Fibonacci case. That is, the implication $L R(\alpha) \Rightarrow \alpha \geq \alpha_{u}^{+}$holds for general choices of the $\left\{V_{j}\right\}$ in the isotropic XY chain.

Let us explain why we call these Lieb-Robinson bounds "anomalous". The usual LR bound is $L R(1)$ and it implies that commutators (and hence also quantummechanical correlations) are small, up to an exponential error, outside of the "light cone" given by $\left|j-j^{\prime}\right| \leq v t$. For $v>0$, this behavior corresponds to ballistic transport and for $v=0$ to dynamical localization.

By contrast, Theorems 2.4 and 2.6 say that the "light-cone" for the Fibonacci $\mathrm{XY}$ chain is "bent" to be the smaller set $\left|j-j^{\prime}\right| \leq v t^{\alpha_{u}^{+}}$with $0<\alpha_{u}^{+}<1$ (see Proposition 2.8 below). In other words, quantum-mechanical correlations spread sub-ballistically for this model and whenever $\alpha_{u}^{+} \neq \frac{1}{2}$ such behavior is commonly referred to as anomalous transport. To our knowledge, these are the first rigorous results establishing anomalous transport in a many-body quantum system. It is also physically appealing that the upper bound on transport at the one-body level $\alpha_{u}^{+}$is precisely what governs transport at the many-body level, but in light of the fact that the XY chain can be mapped to non-interacting particles, this is not completely surprising either.

Roughly speaking, the anomalous behavior of the Fibonacci XY chain is a consequence of the quasi-periodicity of the $V_{i}$, which is situated in between the two extreme cases of

(a) periodic external fields of $V_{i}$. These correspond to ballistic transport, which is obvious e.g. for the free case $V_{i} \equiv 0$. This case is discussed in an upcoming paper of three of the authors [5].

(b) disordered, that is i.i.d. random, external fields of $V_{i}$. These were proved to lead to zero-velocity LR bounds (i.e. dynamical localization) in [12].

To close the discussion of anomalous transport, we record some known upper and lower bounds on $\alpha_{u}^{+}$.

Proposition 2.8. $\quad$ (i) For all $\lambda>0$, we have $\alpha_{u}^{+}(\lambda)>0$.

(ii) For all $\lambda>\sqrt{24}$, we have

$$
\alpha_{u}^{+}(\lambda) \geq \frac{2 \log \phi}{\log (2 \lambda+22)}
$$

with $\phi=\frac{1+\sqrt{5}}{2}$. As a consequence, $\alpha_{u}^{+}(\lambda)>0.5$ for all $\lambda \leq 4,000$ and so transport is truly anomalous for such $\lambda$.

(iii) For all $\lambda \geq 8$, we have $\alpha_{u}^{+}(\lambda)<0.88$.

Proof. For (i), see [4] and note that $\tilde{\alpha}_{u}^{+} \leq \alpha_{u}^{+}$according to Lemma 7.2. For (ii), we invoke Theorem 3 of [7] and use $\tilde{\alpha}_{u}^{+} \leq \alpha_{u}^{+}$again. For (iii), we use the upper bound established in Theorem 3 of [6]

$$
\alpha_{u}^{+} \leq \frac{2 \log \phi}{\log \xi(\lambda)},
$$


for $\lambda \geq 8$ and $\xi(\lambda)=\frac{1}{2}\left(\lambda-4+\sqrt{(\lambda-4)^{2}-12}\right.$. Since $\lambda \mapsto \xi(\lambda)$ is monotone increasing with $\xi(8)=3$, we get

$$
\alpha_{u}^{+}(\lambda) \leq \frac{2 \log \phi}{\log 3} \approx 0.876
$$

for all $\lambda \geq 8$.

2.2. Equality of Transport Exponents. We explicitly note the following corollary of the proof of Proposition 5.1, because we believe it to be of independent interest. The averaged transport exponents $\tilde{\alpha}_{u}^{ \pm}$are defined in Section 7 .

Corollary 2.9. Let $\lambda>0$. Then, $\alpha_{u}^{+}=\tilde{\alpha}_{u}^{+}=\tilde{\alpha}_{u}^{-}$

The second equality was already observed in [3], the first one is new.

\section{The Relation to One-Body Dynamics}

3.1. Diagonalizing the XY Chain. We will use the standard procedure, going back to [18], of diagonalizing the XY chain via the Jordan-Wigner transformation to free fermions, followed by a Bogoliubov transformation.

We only recall what we need to establish notation for the relevant objects. For the details of the diagonalization procedure, we refer to Section 3.1 in [12]. The first step is to introduce the lowering operator

$$
a_{j}=\frac{1}{2}\left(\sigma_{j}^{x}-i \sigma_{j}^{y}\right)
$$

and its adjoint the raising operator $a_{j}^{*}$ for all $1 \leq j \leq n$. The Jordan-Wigner transformation maps these to the fermion operators ${ }^{2}$

$$
c_{1}=a_{1}, \quad c_{j}=\sigma_{1}^{z} \ldots \sigma_{j-1}^{z} a_{j} \quad \text { for } 2 \leq j \leq n .
$$

In terms of these operators, the Hamiltonian reads

$$
H_{n}^{X Y}=\sum_{j=1}^{n-1} \sum_{k=1}^{n} c_{j}^{*} M_{j, k} c_{k}
$$

where we introduced the $2 \times 2$ block matrix

$$
M=\left(\begin{array}{cc}
H_{n} & 0 \\
0 & -H_{n}
\end{array}\right)
$$

which features the $n \times n$ matrix

$$
H_{n}=\left(\begin{array}{cccc}
V_{1} & 1 & & \\
1 & \ddots & \ddots & \\
& \ddots & \ddots & 1 \\
& & 1 & V_{n}
\end{array}\right) .
$$

We will refer to $H_{n}$ as the one-body Fibonacci Hamiltonian. To emphasize that it depends on the phase $\omega \in[0,1)$ we sometimes write $H_{n}(\omega)$.

We will heavily use that the Heisenberg dynamics of the $c_{j}$ operators is given in the following simple fashion.

\footnotetext{
${ }^{2}$ This means that they satisfy the canonical anticommutation relations.
} 
Proposition $3.1([12])$. For all $1 \leq j, k \leq n$ and all $\omega \in[0,1)$, we have

$$
\tau_{t}^{n}\left(c_{j}\right)=\sum_{k=0}^{n}\left(e^{-2 i H_{n}(\omega) t}\right)_{j, k} c_{k} .
$$

Proof. This is a consequence of formula (3.15) in [12] for $\tau_{t}^{n}\left(c_{j}\right)$, which is proved via Bogoliubov transformation, and the observation that $\left(e^{-2 i M t}\right)_{j, n+k}=0$ for all $0 \leq j \leq n$ due to the block diagonal structure of $M$.

3.2. Relating LR Bounds for the XY Chain to Fermionic LR Bounds. The following lemma is instrumental in relating the LR bounds for the $c_{j}$ back to LR bounds for local observables in the XY chain. The difficulty is that the JordanWigner transformation (4) is non-local. This was overcome in [12] at the (small) price of the extra sum over $k$ in (8).

Definition 3.2. We say that $L R_{\text {fermi }}(\alpha)$ holds if there exist non-negative constants $C_{1}, \mu, v$ such that for all integers $1 \leq j<j^{\prime} \leq n$ and all $t>0$, we have

$$
\left\|\left[\tau_{t}^{n}\left(c_{j}\right), B\right]\right\|+\left\|\left[\tau_{t}^{n}\left(c_{j}^{*}\right), B\right]\right\| \leq C_{1}\|B\| e^{-\mu\left(\left|j^{\prime}-j\right|-v t^{\alpha}\right)}
$$

for all $B \in \mathcal{A}_{\left\{j^{\prime}, \ldots, n\right\}}$.

For obvious reasons, we will make heavy use of

Lemma 3.3 ([12]). LR( $\alpha)$ holds if and only if $L R_{\mathrm{fermi}}(\alpha)$ holds.

We use the strategy of the proof of Theorem 3.2 in [12], but we allow for a $t$-dependence of the form appearing on the right-hand side of the anomalous LR bound and we note that the argument can also be run in reverse.

Proof. We first prove the "if" part. Let $A=a_{j}$. Since $\left(\sigma_{i}^{z}\right)^{2}=1$ and $\left[\sigma_{i}^{z}, \sigma_{j}^{z}\right]=0$ when $i \neq j$, we can easily invert the Jordan-Wigner transformation (4) to get

$$
a_{1}=c_{1}, \quad a_{j}=\sigma_{1}^{z} \ldots \sigma_{j-1}^{z} c_{j}, \quad \forall j \geq 2 .
$$

By the automorphism property of $\tau_{t}^{n}$ and the "Leibniz rule" for commutators

$$
[A B, C]=A[B, C]+[A, C] B,
$$

we have

$$
\left[\tau_{t}^{n}\left(a_{j}\right), B\right]=\tau_{t}^{n}\left(\sigma_{1}^{z}\right) \ldots \tau_{t}^{n}\left(\sigma_{j-1}^{z}\right)\left[\tau_{t}^{n}\left(\sigma_{j}^{z}\right), B\right]+\left[\tau_{t}^{n}\left(\sigma_{1}^{z}\right), \ldots, \tau_{t}^{n}\left(\sigma_{j-1}^{z}\right), B\right] \tau_{t}^{n}\left(c_{j}\right) .
$$

By unitarity of $\tau_{t}^{n}$, this implies

$$
\left\|\left[\tau_{t}^{n}\left(a_{j}\right), B\right]\right\| \leq\left\|\left[\tau_{t}^{n}\left(c_{j}\right), B\right]\right\|+C(j-1, B),
$$

where we introduced

$$
C(l, B)=\left\|\left[\tau_{t}^{n}\left(\sigma_{1}^{z}\right), \ldots, \tau_{t}^{n}\left(\sigma_{l}^{z}\right), B\right]\right\| .
$$

Applying (7) again, we find

$$
C(l, B) \leq C(l-1, B)+\left\|\left[\tau_{t}^{n}\left(\sigma_{l}^{z}\right), B\right]\right\| .
$$

Since $\sigma_{l}^{z}=2 c_{l}^{*} c_{l}-\mathbb{1}_{\mathbb{C}^{2}}$, we get

$$
C(l, B) \leq C(l-1, B)+2\left\|\left[\tau_{t}^{n}\left(c_{l}\right), B\right]\right\|+2\left\|\left[\tau_{t}^{n}\left(c_{l}^{*}\right), B\right]\right\|,
$$

which we can iterate to obtain

$$
\left\|\left[\tau_{t}^{n}\left(a_{j}\right), B\right]\right\| \leq \sum_{l=1}^{j}\left(\left\|\left[\tau_{t}^{n}\left(c_{l}\right), B\right]\right\|+\left\|\left[\tau_{t}^{n}\left(c_{l}^{*}\right), B\right]\right\|\right) .
$$


By performing a geometric series, it is now obvious that $L R_{\text {fermi }}(\alpha)$ implies $L R(\alpha)$ in the special case when $A=a_{j}$. Extending this to all of $\mathcal{A}_{j}$, which is spanned by $\left\{a_{j}, a_{j}^{*}, a_{j}^{*} a_{j}, a_{j} a_{j}^{*}\right\}$, is not hard; we refer to [12] for the details.

The "only if" part follows by the exact same reasoning, since the Jordan-Wigner transform and its inverse are of the same form.

\section{Proof of the Second Main Result}

We begin with the proof of the second main result, Theorem 2.6.

Lemma 4.1. For any $l \in[1, n)$ and any $r \in(l, n]$, for any $t \in \mathbb{R}$,

$$
\left\|\left[\tau_{t}^{n}\left(c_{l}\right), a_{r}^{*}\right]\right\| \geq\left|\left(e^{-2 i H_{n}(\omega) t}\right)_{l, r}\right| .
$$

Proof. From (6) we have

$$
\left\|\left[\tau_{t}^{n}\left(c_{l}\right), a_{r}^{*}\right]\right\|=\left\|\left[\sum_{j=1}^{n}\left(e^{-2 i H_{n}(\omega) t}\right)_{l, j} c_{j}, a_{r}^{*}\right]\right\| .
$$

Observe that for all $j<r, a_{r}^{*}$ commutes with $c_{j}$. Thus we can write

$$
\left\|\left[\tau_{t}^{n}\left(c_{l}\right), a_{r}^{*}\right]\right\|=\left\|\left[\sum_{j \geq r}^{n}\left(e^{-2 i H_{n}(\omega) t}\right)_{l, j} c_{j}, a_{r}^{*}\right]\right\| .
$$

Notice that for each $j>r, c_{j}=\sigma_{1}^{(z)} \cdots \sigma_{j-1}^{(z)} a_{j}$, and $a_{r}^{*}$ commutes with $a_{j}$ and every $\sigma_{i}^{(z)}$ with $i \neq r$. On the other hand, notice that for every $i, \sigma_{i}^{(z)} v=v$ with $v=\bigotimes_{1}^{n}\left(\begin{array}{l}1 \\ 0\end{array}\right)$. Thus, since $a_{r}^{*}\left(\begin{array}{l}1 \\ 0\end{array}\right)=0$, we have

$$
\left(\left[\sum_{j \geq r+1}^{n}\left(e^{-2 i H_{n}(\omega) t}\right)_{l, j} c_{j}, a_{r}^{*}\right]\right) v=0,
$$

and

$$
\left[\left(e^{-2 i H_{n}(\omega) t}\right)_{l, r} c_{r}, a_{r}^{*}\right] v=\left(e^{-2 i H_{n}(\omega) t}\right)_{l, r} v .
$$

Thus we have

$$
\left\|\left[\tau_{t}^{n}\left(c_{l}\right), a_{r}^{*}\right]\right\| \geq\left\|\left[\left(e^{-2 i H_{n}(\omega) t}\right)_{l, r} c_{r}, a_{r}^{*}\right] v\right\|=\left\|\left(e^{-2 i H_{n}(\omega) t}\right)_{l, r} v\right\|=\left|\left(e^{-2 i H_{n}(\omega) t}\right)_{l, r}\right| .
$$

Proof of Theorem 2.6. By the previous lemma and the assumption of Theorem 2.6, we have

$$
\left|\left\langle\delta_{l}, e^{-2 i t H_{n}(\omega)} \delta_{r}\right\rangle\right| \leq\left\|\left[\tau_{t}^{n}\left(c_{l}\right), a_{r}^{*}\right]\right\| \leq C e^{-\eta\left(r-l-v|t|^{\alpha}\right)} .
$$

Let us shift everything so that we get

$$
\left|\left\langle\delta_{l}, e^{-2 i t H_{n}(\omega)} \delta_{r}\right\rangle\right|=\left|\left\langle\delta_{r-l}, e^{-2 i t H_{n}(\tilde{\omega})} \delta_{0}\right\rangle\right|
$$

(notice the change in the phase from $\omega$ to $\tilde{\omega}$ as a result of the shift). Since we initially put no restrictions on $l$ and $r$, we get, for each $m$,

$$
\left|\left\langle\delta_{m}, e^{-2 i t H_{n}(\tilde{\omega})} \delta_{0}\right\rangle\right|^{2} \leq C e^{-\eta\left(m-v|t|^{\alpha}\right)} .
$$


Now if we define, for all $N \leq n$,

$$
P_{\text {out }}^{(n)}(N, t):=\sum_{n \geq m \geq N}\left|\left\langle\delta_{m}, e^{-i t H_{n}(\tilde{\omega})} \delta_{0}\right\rangle\right|^{2},
$$

and set $P_{\text {out }}^{(n)}(N, t)=0$ for all $N>n$, we obtain

$$
P_{\text {out }}^{(n)}(N, 2 t) \leq \sum_{n \geq m \geq N} C e^{-\eta\left(m-v|t|^{\alpha}\right)} \lesssim e^{-\eta\left(N-v|t|^{\alpha}\right)} .
$$

By the dominated convergence theorem we get

$$
\lim _{n \rightarrow \infty} P_{\text {out }}^{(n)}(N, 2 t)=P(N-1,2 t) \text { for all } t .
$$

In particular,

$$
P\left(t^{\gamma}-1, t\right) \lesssim e^{-\eta\left(t^{\gamma}-v|t / 2|^{\alpha}\right)} .
$$

Thus for all $\gamma>\alpha$, we have $P\left(t^{\gamma}-1, t\right) \lesssim e^{-(\eta / 2) t^{\gamma}}$ for $t \gg 1$. By (2) and (3), this implies $\gamma \geq \alpha_{u}^{+}$, so that $\alpha \geq \alpha_{u}^{+}$.

\section{Proof of the First Main Result}

5.1. The Transport Exponent in a New Guise. We begin the proof of the first main result, Theorem 2.4. It will be convenient for us to use the following quantity, which we will soon see agrees with $\alpha_{u}^{+}$, introduced in the recent paper [3] to study transport exponents from a dynamical systems perspective.

We define the following quantity

$$
\alpha^{\prime} \equiv \alpha^{\prime}(\lambda)=\frac{\log \left(\frac{1+\sqrt{5}}{2}\right)}{\lim _{k \rightarrow \infty} \frac{1}{k} \log \min _{j=1, \ldots F_{k}}\left|x_{k}^{\prime}\left(E_{k}^{j}\right)\right|},
$$

where $F_{k}$ the $k$-th Fibonacci number, the Fibonacci trace map $x_{k}$ is defined in (28) and $E_{k}^{(j)}$ is defined in [3]. The precise definitions of these quantities are of limited relevance here, because we will use results of [3] tailor-made for the analysis of $\alpha^{\prime}$ as a "black box". The limit in (10) exists, by Proposition 3.7 in [3].

Another reason why the quantities appearing in the definition of $\alpha^{\prime}$ are of limited relevance here, is that we will prove

Proposition 5.1. It holds that $\alpha^{\prime}=\alpha_{u}^{+}$.

5.2. Key Result: Fermionic LR Bounds from One-Body Dynamics. According to Lemma 3.3, it suffices to prove LR bounds for the fermion operators $c_{j}$. They are established by the following key result:

Theorem 5.2 (Key result). Let $\lambda>0$. If $\alpha>\alpha^{\prime}(\lambda)$, then $L R_{\text {fermi }}\left(\alpha^{\prime}\right)$.

The first main result now follows easily:

Proof of Theorem 2.4. This is a direct consequence of Theorem 5.2, Proposition 5.1 and Lemma 3.3.

It thus remains for us to prove Theorem 5.2 (Section 6) and Proposition 5.1 (Section 7). 


\section{Proof of the Key Result}

6.1. Strategy of Proof. The proof of Theorem 5.2 is based on two main ingredients:

(a) Proposition 3.1, which gives a simple expression for the Heisenberg dynamics of $c_{j}$ in terms of the one-body Fibonacci Hamiltonian $H_{n}$. We recall that the reason why this useful formula holds is that the fermions described by the $c_{j}$ are non-interacting.

(b) Dynamical upper bounds for $H_{n}$, established by Damanik - Tcheremchantsev [6, 7, 2] and Damanik - Gorodetski - Yessen [3]. Some of their tools, like the Dunford functional calculus approach, work for rather general onedimensional quantum systems. However, the crucial exponential lower bound on transfer matrix norms, which is a result of [3] quoted here as Proposition 6.5 is special to the Fibonacci case. Since the methods of [3] apply to arbitrary coupling strength $\lambda>0$, so do our results.

Applying (a) is trivial. Regarding (b), we need to modify the existing arguments somewhat. The main difficulty for us is that bounds on transport exponents involve probabilities (see the definition of $\alpha_{u}^{+}$), which according to quantum theory are given as appropriate $\ell^{2}$-norms. In proving LR bounds however, we are naturally led to consider $\ell^{1}$-norms instead. Since this means we do not have orthogonality at our disposal, we need to develop an alternative approach ${ }^{3}$ and we find that combining a resolution of the identity with Combes-Thomas estimates works.

Some more minor obstructions are:

(i) Our $H_{n}$ is initially only defined on $\mathcal{H}_{n}$, whereas one usually works with full-line or half-line operators. Thus, we first need to extend $H_{n}$ to an appropriate half-line operator $H$ on $\ell^{2}\left(\mathbb{Z}_{+}\right)$.

(ii) To analyze transport exponents, one is only interested in large-time behavior and hence assumes $t \geq 1$ throughout for technical reasons, while we need results for all $t>0$.

(iii) To simplify our analysis, we reduce to the initial state $\delta_{1}=(1,0, \ldots)$ with an arbitrary phase $\omega \in[0,1)$ via the covariance under shifts of the half-line Fibonacci Hamiltonian, see (23). While it is often assumed that $\omega=0$, it is well known, see [6], [3], that one can extend to arbitrary phases $\omega \in[0,1)$ via the methods of [2] and this is precisely what we do.

6.2. The Dunford Functional Calculus Approach to Quantum Dynamics. It suffices to bound $\left[\tau_{t}^{n}\left(c_{j}\right), B\right]$, since one obtains the same bound for $\left[\tau_{t}^{n}\left(c_{j}^{*}\right), B\right]$ by taking adjoints. By $(6)$ and the fact that $\left[c_{k}, B\right]=0$ when $k<j^{\prime}$, we get

$$
\left[\tau_{t}^{n}\left(c_{j}\right), B\right]=\sum_{k=j^{\prime}}^{n}\left(\left(e^{-2 i H_{n}(\omega) t}\right)_{j, k}\left[c_{k}, B\right]\right),
$$

which implies

$$
\left\|\left[\tau_{t}^{n}\left(c_{j}\right), B\right]\right\| \leq 2\|B\| \sum_{k=j^{\prime}}^{n}\left|\left(e^{-2 i H_{n}(\omega) t}\right)_{j, k}\right| .
$$

\footnotetext{
${ }^{3}$ We make the trivial remark that using the Cauchy-Schwarz inequality to go from $\ell^{1}$-norms to $\ell^{2}$-norms, one picks up a factor $\sqrt{n}$ and hence loses the required uniformity in $n$.
} 
From now on, we will work on the half-line Hilbert space

$$
\mathcal{H}=\ell^{2}\left(\mathbb{Z}_{+}\right),
$$

to which we trivially extend $H_{n}$ by setting $H_{n} \delta_{l}=0$ for $l>n$. In the canonical basis $\left\{\delta_{l}\right\}_{l \geq 1}$ of $\mathcal{H}$, we can write the matrix elements of the time-evolution operator as

$$
\left\langle\delta_{j}, e^{-2 i H_{n}(\omega) t} \delta_{k}\right\rangle=\left(e^{-2 i H_{n}(\omega) t}\right)_{j, k} .
$$

We follow the approach of [7] and establish dynamical upper bounds without timeaveraging via the Dunford functional calculus [9]: For all $1 \leq j, k \leq n$, we have

$$
\left\langle\delta_{j}, e^{-2 i H_{n} t} \delta_{k}\right\rangle=-\frac{1}{2 \pi i} \int_{\Gamma} e^{-i t z}\left\langle\delta_{j}, \frac{1}{-2 H_{n}-z} \delta_{k}\right\rangle \mathrm{d} z,
$$

where $z$ stands for $z \mathbb{1}_{\mathbb{Z}_{+}}$and $\Gamma$ is any positively oriented contour in $\mathbb{C}$ that encloses the spectrum $\sigma\left(-2 H_{n}\right)$. Note the slightly unconventional appearance of $-2 H_{n}$ instead of $H_{n}$ on the right-hand side. We will choose the same rectangular $\Gamma$ as was used in the proof of Lemma 2 in [7]. We choose

$$
K=\min \{4,2 \lambda+5\}
$$

and observe

$$
\sigma\left(-2 H_{n}\right) \subset[-K+1, K-1] .
$$

We recall the well-known

Proposition 6.1 (Combes-Thomas estimate [1]). There is a universal constant $C_{T}$ such that for all $1 \leq l, m \leq n$,

$$
\left|\left\langle\delta_{l}, \frac{1}{-2 H_{n}-z} \delta_{m}\right\rangle\right| \leq 2 d^{-1} e^{-C_{T} d|l-m|},
$$

where

$$
d=\min \left\{\operatorname{dist}\left(z, \sigma\left(-2 H_{n}\right)\right), 1\right\} .
$$

Proof. See the appendix in [11] for a few-line proof, which also directly extends to the case considered here where the discrete Laplacian is restricted to a box (this extension was already explicitly observed in [17] for real $z$ ).

When $t \geq 1$, we follow the proof of Lemma 2 in [7] word-for-word until the last step, where we do not use the Cauchy-Schwarz inequality. When $t<1$, we replace $t^{-1}$ by 1 everywhere in the proof, in particular in the definition of the contour $\Gamma$. Note that $\left|e^{-i t z}\right| \leq e$ is still uniformly bounded for all $z \in \Gamma$. The upshot is that (12) yields the estimate

$$
\left|\left\langle\delta_{j}, e^{-2 i H_{n} t} \delta_{k}\right\rangle\right| \leq C e^{-C_{T}(k-j)}+C^{\prime} \int_{-K}^{K}\left|\left\langle\delta_{j}, \frac{1}{-2 H_{n}-E-i \varepsilon} \delta_{k}\right\rangle\right| \mathrm{d} E,
$$

for all $1 \leq j<j^{\prime} \leq k \leq n$. Here, we introduced the quantity

$$
\varepsilon=\max \left\{t^{-1}, 1\right\} \text {, }
$$

which of course satisfies $\varepsilon \leq 1$ for all $t>0$. Using (15) on (11) and performing a geometric series in the first term, we obtain

$$
\left\|\left[\tau_{t}^{n}\left(c_{j}\right), B\right]\right\| \leq C e^{-C_{T}\left(j^{\prime}-j\right)}+C^{\prime} \int_{-K}^{K} \sum_{k=j^{\prime}}^{n}\left|\left\langle\delta_{j}, \frac{1}{-2 H_{n}-E-i \varepsilon} \delta_{k}\right\rangle\right| \mathrm{d} E .
$$

Clearly, we can ignore the first term in the following. 
6.3. Extension to the Half-Line Fibonacci Hamiltonian. By expressing the time evolution in terms of resolvents, we can start modifying $H_{n}$ via the resolvent identity. The effect of these changes will be controlled by combining a resolution of the identity and Combes-Thomas estimates.

We define the half-line operator $H$ on $\ell^{2}\left(\mathbb{Z}_{+}\right)$as the tri-diagonal half-infinite matrix

$$
H=\left(\begin{array}{ccc}
V_{1} & 1 & \\
1 & V_{2} & \ddots \\
& \ddots & \ddots
\end{array}\right)
$$

We denote $R(z)=(-2 H-z)^{-1}$ and $R_{n}(z)=\left(-2 H_{n}-z\right)^{-1}$ with $z=E+i \varepsilon$. We recall the resolvent identity,

$$
R_{n}(z)=R(z)+R(z) 2\left(H_{n}-H\right) R_{n}(z) .
$$

Introducing a resolution of the identity $\sum_{l=1}^{\infty}\left|\delta_{l}\right\rangle\left\langle\delta_{l}\right|$, we can bound the sum in (17) by

$$
\sum_{k=j^{\prime}}^{n}\left|\left\langle\delta_{j}, R_{n}(z) \delta_{k}\right\rangle\right| \leq \sum_{k=j^{\prime}}^{n}\left|\left\langle\delta_{j}, R(z) \delta_{k}\right\rangle\right|+\sum_{l=1}^{\infty}\left|\left\langle\delta_{j}, R(z) \delta_{l}\right\rangle\right| \sum_{k=j^{\prime}}^{n}\left|\left\langle\delta_{l}, 2\left(H_{n}-H\right) R_{n}(z) \delta_{k}\right\rangle\right| .
$$

An important observation is that, on the one hand

$$
H-H_{n}=\chi_{n} H \chi_{n}+\left|\delta_{n}\right\rangle\left\langle\delta_{n+1}|+| \delta_{n+1}\right\rangle\left\langle\delta_{n}\right|,
$$

where we used Dirac notation and wrote $\chi_{n}$ for the indicator function of $\mathbb{Z}_{+} \backslash$ $\{1, \ldots, n\}$. On the other hand, for $j^{\prime} \leq k \leq n, R_{n}(z) \delta_{k}$ is supported in $\mathcal{H}_{n}$ due to the block diagonal structure of $H_{n}-z \mathbb{1}_{\mathbb{Z}_{+}}$. Together, these imply that the only contribution to the $l$-sum in (19) comes from the $l=n+1$ term. Hence,

$$
\sum_{k=j^{\prime}}^{n}\left|\left\langle\delta_{j}, R_{n}(z) \delta_{k}\right\rangle\right| \leq \sum_{k=j^{\prime}}^{n}\left|\left\langle\delta_{j}, R(z) \delta_{k}\right\rangle\right|+2\left|\left\langle\delta_{j}, R(z) \delta_{n+1}\right\rangle\right| \sum_{k=j^{\prime}}^{n}\left|\left\langle\delta_{n}, R_{n}(z) \delta_{k}\right\rangle\right| .
$$

We apply the Combes-Thomas estimate (14) to (20) and grossly over-estimate to get

$$
\begin{aligned}
\sum_{k=j^{\prime}}^{n}\left|\left\langle\delta_{j}, R_{n}(z) \delta_{k}\right\rangle\right| & \leq 2 \sum_{k=j^{\prime}}^{n+1}\left|\left\langle\delta_{j}, R(z) \delta_{k}\right\rangle\right|\left(1+2 \varepsilon^{-1} \sum_{k=j^{\prime}}^{n} e^{-C_{T} \varepsilon|k-n|}\right) \\
& \leq C \frac{1}{\varepsilon\left(1-e^{-C_{T} \varepsilon}\right)} \sum_{k=j^{\prime}}^{\infty}\left|\left\langle\delta_{j}, R(z) \delta_{k}\right\rangle\right| .
\end{aligned}
$$

Note that $n$ has disappeared from the last expression. Since we are aiming for uniformity in $n$, this is un-problematic. Using (21) to estimate (17) and recalling $z=E+i \varepsilon$, we see that it remains to control

$$
C \frac{1}{\varepsilon\left(1-e^{-C_{1} \varepsilon}\right)} \int_{-K}^{K} \sum_{k=j^{\prime}}^{\infty}\left|\left\langle\delta_{j}, R(E+i \varepsilon) \delta_{k}\right\rangle\right| \mathrm{d} E
$$


6.4. Covariance of $H(\omega)$ under Shifts. For $l \geq 0$, let $T_{l}$ be the right-shift operator defined by

$$
T_{l} \delta_{m}=\delta_{m+l}
$$

with adjoint operator given by the left-shift $T_{l}^{*}=T_{-l}$. We observe the following covariance property of $H(\omega)$ :

$$
T_{l}^{*} H(\omega) T_{l}=H\left(\omega_{l}\right)
$$

where

$$
\omega_{l}=\omega+l \phi^{-1} \bmod 1 .
$$

Hence, for all $1 \leq j<j^{\prime}$, functional calculus implies

$$
\begin{aligned}
\sum_{k=j^{\prime}}^{\infty}\left|\left\langle\delta_{j}, R(E+i \epsilon, \omega) \delta_{k}\right\rangle\right| & =\sum_{k=j^{\prime}}^{\infty}\left|\left\langle\delta_{1}, T_{j-1}^{*} R(E+i \epsilon, \omega) T_{j-1} \delta_{1+k-j}\right\rangle\right| \\
& \leq \sum_{k=0}^{\infty} \sup _{\omega \in[0,1)}\left|\left\langle\delta_{1}, R(E+i \epsilon, \omega) \delta_{k+N}\right\rangle\right|,
\end{aligned}
$$

where we wrote $R(z, \omega)=(-2 H(\omega)-z)^{-1}$ for emphasis and introduced the integer

$$
N \equiv 1+j^{\prime}-j
$$

for notational convenience.

6.5. Bounding Resolvent Matrix Elements by Transfer Matrix Norms. The next step is to bound matrix elements of the resolvent in terms of transfer matrix norms, following the strategy of Theorem 7 in [6] of comparing with solutions $u$ to the equation $H u=z u$.

Lemma $6.2([6])$. There exists a constant $C_{2}>0$ such that for all $E \in[-K, K]$ and all $N \geq 3$, we have

$$
\sum_{k=0}^{\infty} \sup _{\omega \in[0,1)}\left|\left\langle\delta_{1}, R(E+i \varepsilon, \omega) \delta_{N+k}\right\rangle\right| \leq C_{2} \frac{1}{\varepsilon\left(1-e^{-C_{T} \varepsilon}\right)} \min _{3 \leq N_{1} \leq N} \sup _{\omega \in[0,1)}\left\|\Phi_{N_{1}}(E+i \varepsilon, \omega)\right\|^{-1} .
$$

Here, $C_{T}$ is the Combes-Thomas constant and $\Phi_{m}(z, \omega)$ is the usual transfer matrix uniquely defined by the requirement that

$$
\left(\begin{array}{c}
u(m+1) \\
u(m)
\end{array}\right)=\Phi_{m}(z, \omega)\left(\begin{array}{c}
u(1) \\
u(0)
\end{array}\right), \quad \forall m \geq 0
$$

holds for every half-infinite vector $u$ satisfying $H(\omega) u=z u$.

Remark 6.3. (i) In [6], the left-hand side of (25) featured an appropriate $\ell^{2}$ norm instead.

(ii) As is emphasized in [6], the proof there only requires that $V$ is real and bounded. Thus, uniformity in $\omega$ comes for free.

(iii) The restriction that $N \geq 3$ is un-problematic for eventually concluding the LR bound, because order one terms can always be absorbed in the constant appearing on its right-hand side. As we will see later, the same idea will allow us to dispose of the potentially large $\varepsilon$-dependent pre-factor introduced by the Combes-Thomas estimates, at the expense of increasing the exponential growth in $t$ on the right-hand side of the LR bound. 
Before the proof, we introduce some notation, which is similar to that in [6]. For $N \geq 3$ and $l \geq 1$, let

$$
V_{m}^{ \pm, N}(\omega)= \begin{cases}V_{m}(\omega), & \text { if } m \leq N \\ \pm 2 K, & \text { if } m \geq N+1\end{cases}
$$

and let $H_{N}^{ \pm}(\omega)$ be the half-infinite matrix obtained from $H(\omega)$ by replacing $V_{m}(\omega)$ with $V_{m}^{ \pm, N}(\omega)$ for all $m \geq 1$. Moreover, denote $R_{N}^{ \pm}(z)=\left(-2 H_{N}^{ \pm}-z\right)^{-1}$. The crucial step is to establish the following analogue to Lemma 1 in [6].

Lemma $6.4([6])$. We have

$$
\begin{aligned}
& \sum_{k=0}^{\infty} \sup _{\omega \in[0,1)}\left|\left\langle\delta_{1}, R(E+i \varepsilon, \omega) \delta_{N+k}\right\rangle\right| \\
& \leq C \frac{1}{\varepsilon\left(1-e^{-C_{T} \varepsilon}\right)} \sum_{k=0}^{\infty} \sup _{\omega \in[0,1)}\left|\left\langle\delta_{1}, R_{N}^{ \pm}(E+i \varepsilon, \omega) \delta_{N+k}\right\rangle\right| .
\end{aligned}
$$

Proof. We use the same combination of resolvent identity, resolution of the identity and Combes-Thomas estimate as in the extension from $H_{n}$ to $H$ before. Let $z=$ $E+i \varepsilon$. By the resolvent identity,

$$
R(z, \omega)=R_{N}^{ \pm}(z, \omega)+R_{N}^{ \pm}(z, \omega) \chi_{N}(V(\omega) \mp 2 K) R(z, \omega),
$$

where $\chi_{N}$ is the indicator function of the set $\mathbb{Z}_{+} \backslash\{1, \ldots, N\}$ and

$$
V(\omega)=\sum_{m \geq 1} V_{m}(\omega)\left|\delta_{m}\right\rangle\left\langle\delta_{m}\right|
$$

We use this on the left-hand side of (26) and introduce a resolution of the identity to get

$$
\begin{aligned}
& \sum_{k=0}^{\infty} \sup _{\omega \in[0,1)}\left|\left\langle\delta_{1}, R(z, \omega) \delta_{N+k}\right\rangle\right| \\
& \leq C \sum_{k=0}^{\infty} \sup _{\omega \in[0,1)}\left(\left|\left\langle\delta_{1}, R_{N}^{ \pm}(z, \omega) \delta_{N+k}\right\rangle\right|\right. \\
&\left.+\sum_{l=0}^{\infty}\left|\left\langle\delta_{1}, R_{N}^{ \pm}(z, \omega) \delta_{N+l}\right\rangle\left\langle\delta_{N+l}, R(z) \delta_{N+k}\right\rangle\right|\right),
\end{aligned}
$$

where we also used that $V(\omega) \mp 2 K$ is bounded, uniformly in $\omega$. By the ordinary Combes-Thomas estimate on the half-line, see (A.11) in [11], we have

$$
\left|\left\langle\delta_{N+l}, R(z) \delta_{N+k}\right\rangle\right| \leq \frac{C}{\varepsilon} e^{-C_{T} \varepsilon|k-l|}
$$

with constants that are uniform in $E, l$ and $\omega$. Using this on (27) and performing a geometric series, we get

$$
\sum_{k=0}^{\infty} \sup _{\omega \in[0,1)}\left|\left\langle\delta_{1}, R(z, \omega) \delta_{N+k}\right\rangle\right| \leq C \frac{1}{\varepsilon\left(1-e^{-C_{T} \varepsilon}\right)} \sum_{k=0}^{\infty} \sup _{\omega \in[0,1)}\left|\left\langle\delta_{1}, R_{N}^{ \pm}(z, \omega) \delta_{N+k}\right\rangle\right|
$$

and we are done. 
Proof of Lemma 6.2. Note that $K$ defined by (13) satisfies $K \geq 4$. We follow wordfor-word the proofs of Lemmas 2 and 3 in [6] and use monotonicity of $\sqrt{\cdot}$ where appropriate. We stress that

(a) these arguments only assume that $V$ is real and bounded and so none of the constants that appear depend on $\omega$;

(b) while the proof of Lemma 2 may appear to be for the whole-line case, due to the $m_{-}(z)$-term in formulae (35) and (36), it also applies to the half-line case with Dirichlet boundary condition (in which case the $m_{-}(z)$ disappears). In fact, [6] note in the introduction that they consider both, the full-line and the half-line case, simultaneously. See also [16], where this method was originally developed.

Together with Lemma 6.4, these arguments imply that for all $N \geq 3$ and all $E \in$ $[-K, K]$, we have

$$
\sum_{k=0}^{\infty} \sup _{\omega}\left|\left\langle\delta_{1}, \frac{1}{(R(z, \omega))} \delta_{N+k}\right\rangle\right| \leq C \frac{1}{\varepsilon\left(1-e^{-C^{\prime} \varepsilon}\right)} \sup _{\omega}\left\|\Phi_{N}(z, \omega)\right\|^{-1},
$$

for all $N \geq 3$. Since the left-hand side is monotone decreasing in $N$, we can take the minimum over $3 \leq N_{1} \leq N$ and we are done.

6.6. Lower Bounds on Transfer Matrix Norms and Conclusion. Lower bounds on transfer matrix norms $\left\|\Phi_{N}(z, \omega)\right\|$ for the Fibonacci Hamiltonian can be obtained by studying the "trace map", a second-order difference equation for the sequence

$$
x_{M} \equiv x_{M}(z, 0) \equiv \operatorname{tr} \frac{1}{2} \Phi_{F_{M}}(z, 0),
$$

where $F_{M}$ denotes the $M$-th Fibonacci number and the transfer matrix $\Phi$ was defined in Lemma 6.2. Bounds on $\left|x_{M}\right|$ lead to bounds on $\left\|\Phi_{F_{M}}(z, 0)\right\|$ via the trivial estimate

$$
\|A\| \geq \frac{1}{2}|\operatorname{tr} A|,
$$

which holds for any $2 \times 2$ matrix $A$. For a detailed exposition of the trace map, we refer to Section 4 of [6]. Here, we use an improved version of the results of [6] established by [3] to prove their Proposition 3.8.

Proposition 6.5 ([3]). Let $\omega=0$. Recall the definitions of $\alpha(\lambda), \xi(\lambda)$ in Theorem 2.4. There exists $\delta>0$ such that for all $\varepsilon \in(0,1]$ and all $E \in[-K, K]$, we have

$$
\left|x_{M}(E+i \varepsilon, 0)\right| \geq(1+\delta)^{F_{M-M_{0}}}
$$

for all $M \geq M_{0}+1$, where $M_{0} \geq K$ is chosen such that

$$
C_{\delta}^{\prime} F_{M_{0}}^{-s^{\prime}}<\varepsilon,
$$

where $s^{\prime}>\alpha^{\prime}$ and $C_{\delta}^{\prime}>0$ is an appropriate constant.

Proof. See the proof of Proposition 3.8 (b) in [3].

We are now ready to give the 
Proof of Theorem 5.2. Recall that $N \equiv 1+j^{\prime}-j$ and recall the definition of $\varepsilon$ in (16). We start from (17), apply (21) to extend to the half-line, (24) to reduce to the case $j=1$ at the price of a $\sup _{\omega}$ and finally we apply Lemma 6.2 to conclude (31)

$$
\begin{aligned}
& \left\|\left[\tau_{t}^{n}\left(c_{j}\right), B\right]\right\| \\
& \leq C\|B\| e^{-C_{T}\left(j^{\prime}-j\right)}+C^{\prime}\|B\| \frac{1}{\varepsilon^{2}\left(1-e^{-C_{T} \varepsilon}\right)} \int_{-K}^{K} \min _{3 \leq N_{1} \leq N} \sup _{\omega \in[0,1)}\left\|\Phi_{N_{1}}(E+i \varepsilon, \omega)\right\|^{-1} \mathrm{~d} E .
\end{aligned}
$$

As remarked before, we can safely ignore the first term, since it gives an LR bound with $v=0$. Moreover, since $\left\|\left[\tau_{t}^{n}\left(c_{j}\right), B\right]\right\| \leq 2\|B\|$ and the claimed LR bound allows for a constant on the right-hand side, we may ignore order-one quantities in the following.

Step 1: We would like to apply Proposition 6.5 together with (29) to bound the transfer matrix norms from below by an exponentially increasing quantity. However, it is assumed in Proposition 6.5 that $\omega=0$, while we require uniformity in $\omega$. In order to extend to general $\omega \in[0,1$ ), we use results of [2] (this possibility was already noted in passing in [6]): According to Proposition 3.4 in [2], we have

$$
x_{M}(E, \omega)=x_{M}(E, 0)
$$

for all $E \in \mathbb{R}$, all $\omega \in[0,1$ ) and either (a) all odd $M$ or (b) all even $M$. Both sides of this equation are complex analytic in $E$, which is obvious from the usual definition of the transfer matrices of positive index, see (12) in [6]. Thus, we can extend the relation to

$$
x_{M}(z, \omega)=x_{M}(z, 0),
$$

with $z$ complex and $M$ as before.

Step 2: We choose $M_{1}^{\prime}$ to be the largest integer such that $F_{M_{1}^{\prime}} \leq N$, i.e. we have

$$
F_{M_{1}^{\prime}} \leq N<F_{M_{1}^{\prime}+1} \text {. }
$$

If it so happens that (32) holds for all even (odd) integers $M$, but $M_{1}^{\prime}$ is odd (even), we set $M_{1} \equiv M_{1}^{\prime}-1$. Otherwise, we set $M_{1} \equiv M_{1}^{\prime}$. We can assume $M_{1} \geq 3$, because $M_{1}<3$ yields an order-one bound on $N=1+j^{\prime}-j$ and such terms can be ignored as we explained before.

Then, we estimate the minimum in (31) by the $M_{1}$-th term and we use (29), (32) and Proposition 6.5 to find, for some $\delta>0$,

$$
\left\|\left[\tau_{t}^{n}\left(c_{j}\right), B\right]\right\| \leq C\|B\| e^{-C_{T}\left(j^{\prime}-j\right)}+C^{\prime}\|B\|\left(\frac{1}{\varepsilon\left(1-e^{-C_{T} \varepsilon}\right)}\right)^{2}(1+\delta)^{-F_{M_{1}-M_{0}}}
$$

if we have $M_{1} \geq M_{0}+1$ with $M_{0}$ chosen minimally, i.e.

$$
C_{\delta}^{\prime} F_{M_{0}}^{-s^{\prime}}<\varepsilon \leq C_{\delta}^{\prime} F_{M_{0}-1}^{-s^{\prime}} .
$$

Next, we will investigate this condition further.

Step 3: The first inequality right above is equivalent to

$$
F_{M_{0}}>\left(\frac{C_{\delta}^{\prime}}{\varepsilon}\right)^{1 / s^{\prime}}
$$

We will use the well-known fact that

$$
\frac{\phi^{l}}{\sqrt{5}}-\frac{1}{2} \leq F_{l} \leq \frac{\phi^{l}}{\sqrt{5}}+\frac{1}{2}
$$


for all $l \geq 0$. It implies that, up to order-one constants, one can replace $F_{l}$ by $\phi^{l} / \sqrt{5}$. Using this and convexity of the exponential function, we conclude that the second term in (33) is bounded by

$$
C\left(\frac{1}{\varepsilon\left(1-e^{-C_{T} \varepsilon}\right)}\right)^{2} e^{-\mu^{\prime}\left(F_{M_{1}}-F_{M_{0}}\right)}
$$

where we introduced the positive quantity

$$
\mu^{\prime}=\frac{2 \log (1+\delta)}{\sqrt{5}} .
$$

We use (35) and recall the definitions of $M_{0}, M_{1}^{\prime}$ as certain minimal/maximal integers to get

$$
\begin{aligned}
& F_{M_{0}} \leq \phi F_{M_{0}-1}-1 \leq \phi\left(\frac{C_{\delta}^{\prime}}{\varepsilon}\right)^{1 / s^{\prime}} \\
& F_{M_{1}} \geq \phi^{-1} F_{M_{1}^{\prime}}+1 \geq \phi^{-2}\left(F_{M_{1}^{\prime}+1}+C\right) \geq \phi^{-2} N+2 .
\end{aligned}
$$

We use these to bound (36) by

$$
C\left(\frac{1}{\varepsilon\left(1-e^{-C_{T} \varepsilon}\right)}\right)^{2} e^{-\mu\left(N-v \varepsilon^{-1 / s^{\prime}}\right)}
$$

with

$$
\mu=\phi^{-2} \mu^{\prime}, \quad v=\phi^{3}\left(C_{\delta}^{\prime}\right)^{1 / s^{\prime}}
$$

and this bound holds for $N \geq v \varepsilon^{-1 / s^{\prime}}$.

Step 4: We come to the conclusion, which mainly involves making order-one changes to accommodate some exceptional cases. Suppose that $t \geq 1$, i.e. $\varepsilon=t^{-1}$ according to the definition of $\varepsilon$ in (16). It is crucial that the pre-factor in (37), which quantifies the cost of our two Combes-Thomas estimates, can be bounded via

$$
\left(\frac{t}{1-e^{-C_{T} t^{-1}}}\right)^{2} \leq C_{T}^{\prime} t^{4}
$$

for all $t \geq 1$, where $C_{T}^{\prime}$ is a universal constant. Moreover, $C_{T}^{\prime} t^{4}$ can be bounded in terms of the exponential increase in $t$ in (37), by an order-one constant in front and a change of $1 / s^{\prime}>\alpha^{\prime}$ to a slightly larger value, but since $1 / s^{\prime}$ may be arbitrarily close to $\alpha^{\prime}$ this change is irrelevant. We have shown that

$$
\left\|\left[\tau_{t}^{n}\left(c_{j}\right), B\right]\right\| \leq C \exp \left(-\mu\left(\left|j^{\prime}-j\right|-v t^{1 / s^{\prime}}\right)\right)
$$

for all $t \geq 1$, all $1 / s^{\prime}>\alpha^{\prime}$, whenever $\left|j^{\prime}-j\right|-v t^{\alpha(\lambda)} \geq 0$. In the case $t<1$, we have $\varepsilon=1$ according to (16) and all occurrences of $t$ in the previous argument can be replaced by order-one quantities. Since the exponential is bounded in $t<1$, we can then re-instate the $t$-dependence by yet another order-one change of the constants. Hence, (39) extends to all $t>0$.

Finally, when $\left|j^{\prime}-j\right|-v t^{1 / s^{\prime}}<0$, the argument of the exponential in the LR bound is positive and hence the entire right-hand side is at least order-one. This finishes the proof. 


\section{Proof that $\alpha^{\prime}=\alpha_{u}^{+}$}

In this final section, we prove Proposition 5.1, which we recall states that

$$
\alpha^{\prime}(\lambda)=\alpha_{u}^{+}(\lambda)
$$

for all $\lambda>0$ (we will suppress $\lambda$ from the notation from now on). The proof will proceed via the following two lemmas. The first one features some other transport exponents and we recall their definitions.

Definition 7.1. We write

$$
|X|^{p}(t)=\sum_{n>0}|n|^{p}\left|\left\langle e^{-i t H} \delta_{1}, \delta_{n}\right\rangle\right|^{2}
$$

for the $p$-th moment of the position operator. For any function $f(t)$, define its time-average by

$$
\langle f\rangle(T)=\frac{2}{T} \int_{0}^{\infty} e^{-2 t / T} f(t) \mathrm{d} t
$$

for all $T>0$. We define the transport exponents

$$
\begin{aligned}
& \beta^{+}(p)=\limsup _{t \rightarrow \infty} \frac{\log |X|^{p}(t)}{p \log t}, \\
& \tilde{\beta}^{+}(p)=\limsup _{t \rightarrow \infty} \frac{\log \left\langle|X|^{p}(t)\right\rangle}{p \log t} .
\end{aligned}
$$

The time-averaged upper transport exponent can be defined by analogy with (2) and (3), or by

$$
\tilde{\alpha}_{u}^{+}=\lim _{p \rightarrow \infty} \tilde{\beta}^{+}(p) .
$$

The two definitions are equivalent; see Theorems 2.18, 2.22 of [8], where it is also shown that

$$
\alpha_{u}^{+}=\lim _{p \rightarrow \infty} \beta^{+}(p) .
$$

Lemma 7.2. We have $\tilde{\alpha}_{u}^{+} \leq \alpha_{u}^{+}$.

Proof. Fix $p \in(0, \infty)$ and take an arbitrary $\gamma>\beta^{+}(p)$. Then

$$
|X|^{p}(t) \leq C t^{p \gamma}
$$

for some $C$ independent of $t$, so the time-averaged $p$-th moment obeys

$$
\left\langle|X|^{p}\right\rangle(T)=\frac{2}{T} \int_{0}^{\infty} e^{-2 t / T}|X|^{p}(t) d t \leq 2 C C_{1} T^{p \gamma},
$$

where $C_{1}=\int_{0}^{\infty} e^{-2 x} x^{p \gamma} d x$. This in turn implies $\tilde{\beta}^{+}(p) \leq \gamma$, which implies

$$
\tilde{\beta}^{+}(p) \leq \beta^{+}(p)
$$

for any $p \in(0, \infty)$. The claim now follows from (41) and (42).

Lemma 7.3. We have $\alpha_{u}^{+} \leq \alpha^{\prime}$.

Proof. This is just a minor modification of arguments in [3], in which one replaces Parseval's identity with Dunford functional calculus formula (12) to obtain the analogue of formula (26) in [3] with $\tilde{\alpha}_{u}^{+}$replaced by $\alpha_{u}^{+}$. More precisely, instead of invoking [6] to bound averaged probabilities, as done on p. 27 of [3], one uses the same formula without averaging derived in Theorem 1 of [7]. 
Then, part (b) of Proposition 3.8 in [3] directly yields the same bound on $\alpha_{u}^{+}$ as the one on $\tilde{\alpha}_{u}^{+}$in part (c) and the existence of the limit is established by [3, Proposition 3.7].

Remark 7.4. The reason why the averaging in [3] can be removed is that one is dealing with upper bounds and thus the triangle inequality for integrals is available to control the possible oscillation in the Dunford functional calculus formula (12).

Proof of Proposition 5.1 and Corollary 2.9. From Lemmas 7.2 and 7.3, we have, for all $\lambda>0$,

$$
\tilde{\alpha}_{u}^{+} \leq \alpha_{u}^{+} \leq \alpha^{\prime}
$$

By Proposition 3.8 (c) and Proposition 3.7 in [3], we also have

$$
\alpha^{\prime} \leq \tilde{\alpha}_{u}^{+}
$$

and we are done.

\section{The General Sturmian Case}

The Fibonacci Hamiltonian is a representative example of the more general class of Schrödinger operators with potentials modulated by Sturmian sequences. It turns out that much of what can be proved about the Fibonacci Hamiltonian can also be extended to the entire Sturmian class. In this section we show how Theorems 2.4 and 2.6 can be extended to the isotropic $X Y$ model with constant interaction and Sturmian field.

Namely, let us consider

$$
V_{j}=\lambda \chi_{[1-\beta, 1)}(j \beta+\omega \bmod 1),
$$

with $\beta \in(0,1) \cap \mathbb{Q}^{c}, \omega \in[0,1)$, and $\lambda>0$. In what follows, the coefficients of the continued fraction expansion for $\beta$ will be denoted by $a_{1}, a_{2}, \ldots$, and we take $a_{0}=0$. For example, with $\beta$ being the inverse of the golden mean (as in the case of the Fibonacci potential), we have $a_{k}=1$ for all $k \in \mathbb{N}$.

Let us define also

$$
\begin{aligned}
& p_{-1}=1, \quad p_{0}=0, \\
& q_{-1}=0, \quad q_{0}=1,
\end{aligned}
$$

and

$$
\begin{aligned}
p_{k+1} & =a_{k+1} p_{k}+p_{k-1}, \\
q_{k+1} & =a_{k+1} q_{k}+q_{k-1} .
\end{aligned}
$$

In what follows, we restrict our attention to those $\beta \in(0,1) \cap \mathbb{Q}^{c}$, for which the condition

$$
D_{\beta}:=\limsup _{k \in \mathbb{N}} \frac{\log q_{k}}{k}<\infty
$$

is satisfied. For example, in the Fibonacci case, $\left\{q_{k}\right\}$ is precisely the Fibonacci sequence, growing like $\left\{\phi^{k}\right\}$, with $\phi$ being the golden mean. The condition (43) means that $\beta$ is best approximated by the rationals $p_{k} / q_{k}$ with the sequence $\left\{q_{k}\right\}$ not growing faster than an exponential. It is known that the set exceptional to this condition is of zero Lebesgue measure. Moreover, it is known that for almost every 
$\beta, D_{\beta}$ is independent of $\beta$, and its explicit value, known as the Khinchin's constant, is given by

$$
D_{\beta}=D_{K}:=\frac{\pi^{2}}{12 \log 2} \approx 2.68545 \ldots
$$

Upper transport bounds for the discrete Schrödinger operator with potential $\left\{V_{j}\right\}$, in the time-averaged regime, were investigated by Marin in [20], following closely [6]. In particular, Marin obtained the following results.

Theorem 8.1 (Theorem 1 in [20]). With $\beta$ and $\left\{V_{j}\right\}$ as above with $\lambda>20$,

$$
\tilde{\alpha}_{u}^{+} \leq \frac{2 D_{\beta}}{\log \left(\frac{\lambda-8}{3}\right)} .
$$

Moreover, if $a_{k} \neq 1$ for every $k$, then

$$
\tilde{\alpha}_{u}^{+} \leq \frac{D_{\beta}}{\log \left(\frac{\lambda-8}{3}\right)} .
$$

Combining Theorem 8.1 with (43), Marin obtained

Corollary 8.2 (Corollary 1 in [20]). For Lebesgue almost every $\beta$,

$$
\tilde{\alpha}_{u}^{+} \leq \frac{2 D_{K}}{\log \left(\frac{\lambda-8}{3}\right)} .
$$

We also have

Corollary 8.3 (Corollary 2 in $[20]$ ). With $\beta$ such that $a_{k}=a, a \neq 1$, for all $k$,

$$
\tilde{\alpha}_{u}^{+} \leq \frac{\log (a+\beta)}{\log \left(\frac{\lambda-8}{3}\right)} .
$$

The analysis in [20] follows closely [6], which leads to the following upper bound on the time-averaged outside probabilities [20].

$$
\begin{aligned}
\tilde{P}(N(T), T) & \lesssim e^{-c N(T)}+T^{3} \int_{-K}^{K}\left(\max _{1 \leq n \leq N(T)}\left\|M_{n}\left(E+i T^{-1}\right)\right\|^{2}\right)^{-1} d E \\
& \lesssim e^{c N(T)}+T^{3}(1+\delta)^{-2 q\lfloor\sqrt{k(T)\rfloor}},
\end{aligned}
$$

where $c>0$ is a universal constant and $K>0$ is such that the spectrum of the Hamiltonian with potential $\left\{V_{j}\right\}$ is contained in $[1-K, K-1]$, and $M_{n}$ is the transfer matrix over $n$ sites. Here $N(T)=q_{k(T)+\lfloor\sqrt{k(T)}\rfloor}, \delta>0$ small, and $k(T)$ is the unique integer satisfying

$$
\frac{q_{k(T)-1}^{\gamma(\lambda)}}{d_{\delta}} \leq T \leq \frac{q_{k(T)}^{\gamma(\lambda)}}{d_{\delta}},
$$

with appropriately chosen $\gamma(\lambda)$ and $d_{\delta}$ (which are discussed in detail in [20]). The main point is that the bound (44), which leads to Theorem 8.1 and its corollaries, is obtained from [6, Theorem 7], which in turn is based on Parseval's formula. After replacing [6, Theorem 7] with [7, Lemma 2] (which is instead based on Dunford functional calculus), and then repeating the derivation in [20] verbatim, we obtain Theorem 8.1 and all its corollaries in the non-time-averaged regime.

Notice that the derivation of (31) above is model-independent. Then, from (31), the desired Lieb-Robinson bound for the Fibonacci model is obtained by bounding 
the norms of the transfer matrices. These bounds, of course, depend on the model (i.e., on the field $\left\{V_{j}\right\}$ ). In what follows, we work with the restriction (43) in mind.

Notice that, due to the same reasons as in the proof of the first main result above (namely [2, Proposition 3.4]), we have been suppressing the phase $\omega \in[0,1)$ from the notation.

The estimates on the transfer matrix norms given in [20] lead to an estimate similar to (33) with $q_{k}$ in place of $F_{k}$ (see equation (11) in [20]). From this point on, one proceeds just as in the proof of Theorem 2.4 following equation (33) above with the following modifications. We use

$$
\alpha:=\frac{1}{\gamma(\lambda)}+\nu \geq \alpha_{u}^{+}
$$

with arbitrarily small $\nu>0$, in place of $\alpha^{\prime}, d_{\delta}^{-1}$ in place of $C_{\delta}^{\prime}, D_{\beta}$ in place of $\phi$, and a suitable $\mathrm{O}(1)$ perturbation in (35) (see the proof of Theorem 1 in [20] for the definitions of $d_{\delta}$ and $\gamma(\lambda)$ ). This establishes the analog of Theorem 2.4.

The analog of Theorem 2.6 holds due to Remark 2.7.

We remark that in case condition (43) fails, the Schrödinger operator may exhibit ballistic transport; [20] provides an example.

\section{Remark on the Random Dimer Model}

We conclude with a brief discussion as to why the method of this paper will not yield anomalous LR bounds of power-law type for the XY chain with "random dimer" external magnetic field. This section is mostly intended for experts and we refer to $[14,15]$ for details, in particular for the precise definition of the random dimer model. The main message is as follows: Consider the sum over fermionic commutators in (8), which comes from the non-locality of the Jordan-Wigner transformation. In the Fibonacci case, the summands were decaying exponentially, so the sum decays also exponentially and we could conclude that $L R_{\text {fermi }}(\alpha)$ implies $L R(\alpha)$, with the same $\alpha$ ! On the other hand, if the fermionic commutators only decay like a power law, as we will see is the case for the random dimer model, the sum in (8) decreases the power-law decay by one and so, as far as our bounds go, the many-body transport is truly faster than the one-body transport on the power-law scale.

The random dimer model, introduced by Dunlap, Wu and Philips [10], is given by a one-dimensional discrete Laplacian together with a random potential which may take only two values $\pm \lambda$ with $\lambda<1$, but these values always appear in pairs. A characteristic feature of this model is that the dimer-to-dimer transfer matrices commute at the so-called "critical energies" $E_{c}= \pm \lambda$ and that in this (non-generic) case the system exhibits non-trivial transport, in contrast to the usual Anderson localization of a one-dimensional disordered quantum system.

More precisely, it follows from $[7,14,15]$ that for the random dimer model, the transport exponent $\beta^{+}(p)$ defined in (40) satisfies

$$
\beta^{+}(p)=\max \left\{0,1-\frac{1}{2 p}\right\}, \quad \forall p>0 .
$$

According to (42), we have

$$
\alpha_{u}^{+}=1
$$

We now consider an XY chain with external magnetic field given by pairs of random dimers $\pm \lambda$. As pointed out in Remark 2.7, the argument that proved Theorem 2.6 
generalizes to this case and so the best $L R(\alpha)$, in the sense of Definition 2.1, that can hold for this model, is $L R(1)$, but $L R(1)$ holds for much more general models anyway [21].

Roughly speaking, $\alpha_{u}^{+}=1$ means that the one-dimensional quantum particle has exponentially small probability to be observed a distance of order $t$ away from its initial location after time $t$ has passed. While the probability of observation is not exponentially small for distances of order $t^{\beta}$ with $0<\beta<1$, it is polynomially small for $\beta$ sufficiently close to 1 , since $\beta^{+}(p)<1$. With this more refined perspective in mind, one could hope to prove an anomalous LR bound of power-law type such as

$$
\mathbb{E}\left\|\left[\tau_{t}^{n}(A), B\right]\right\| \leq C_{1}\left(\frac{t^{\beta}}{\left|j-j^{\prime}\right|}\right)^{\mu},
$$

for the random dimer model. Here, the objects $A, B, n, j, j^{\prime}, C, \mu$ are chosen as in Theorem 2.4, now of course for the random dimer model, $\mathbb{E}$ denotes the expectation over the randomness and $\beta>0$ should be related to $\beta^{+}(p)$ in some way. Let us now argue why the Jordan-Wigner method will not give such a bound with $\beta<1$.

Following our argument for the Fibonacci case, one first proves a fermionic LR bound of power-law type. Adapting the arguments of [14] to our purposes (the main challenge again being to go from $\ell^{2}$-norms to $\ell^{1}$-norms), one finds

$$
\mathbb{E}\left\|\left[\tau_{n}^{t}\left(c_{j}\right), B\right]\right\|+\mathbb{E}\left\|\left[\tau_{n}^{t}\left(c_{j}^{*}\right), B\right]\right\| \leq C_{1}\left(\frac{t^{\beta^{+}(p)+1 / p+\nu}}{\left|j-j^{\prime}\right|}\right)^{p}
$$

for any $\nu>0$. While it is conceivable that the extra $1 / p$ term in the exponent is technical and can be removed, one still has the following problem: To obtain the LR bound for the corresponding XY chain, one has to take a sum over fermionic LR bounds, see (8). This yields, even without the $1 / p$ term,

$$
C_{1} t^{p \beta^{+}(p)+\nu^{\prime}} \sum_{l=1}^{j}\left(\frac{1}{l+\left|j^{\prime}-j\right|}\right)^{p} \leq C_{1}^{\prime} \frac{t^{p \beta^{+}(p)+\nu^{\prime}}}{\left|j^{\prime}-j\right|^{p-1}}
$$

for any $\nu^{\prime}>0$ and any $p>1$ (for $p \leq 1$, the sum diverges). Recalling (45), we see that $p \beta^{+}(p)=p-1 / 2$ for $p>1$. Hence, the right-hand side above reads

$$
C_{1}^{\prime}\left(\frac{t^{\frac{p-1 / 2}{p-1}+\nu^{\prime \prime}}}{\left|j^{\prime}-j\right|}\right)^{p-1}
$$

for any $\nu^{\prime \prime}>0$. Of course,

$$
\frac{p-1 / 2}{p-1}>1
$$

and so this does not yield an anomalous LR bound (46) with $\beta<1 .{ }^{4}$ In summary, we have seen that the anomalous one-body transport of the random dimer model is still too fast to "survive" the summation (8) that arises from the non-locality of the Jordan-Wigner transformation and hence too fast to yield an anomalous LR bound of power-law type on the many-body level.

\footnotetext{
${ }^{4}$ Note that for $\beta \geq 1$, the LR bound of power-law type (47) is weaker than $L R(1)$, which always holds and yields an exponentially small error term.
} 


\section{REFERENCES}

1. J.M. Combes and L. Thomas, Asymptotic behaviour of eigenfunctions for multiparticle Schrödinger operators, Comm. Math. Phys. 34 (1973), 251-270.

2. D. Damanik, Dynamical upper bounds for one-dimensional quasicrystals, J. Math. Anal. Appl. 303 (2005), 327-341.

3. D. Damanik, A. Gorodetski, and W. Yessen, The Fibonacci Hamiltonian, arXiv:1403.7823.

4. D. Damanik, R. Killip, and D. Lenz, Uniform spectral properties of one-dimensional quasicrystals. III. $\alpha$-continuity, Comm. Math. Phys. 212 (2000), 191-204.

5. D. Damanik, M. Lukic, and W. Yessen, Quantum dynamics of periodic and limit-periodic Jacobi and block Jacobi matrices with applications to some quantum many body problems, preprint.

6. D. Damanik and S. Tcheremchantsev, Upper bounds in quantum dynamics, J. Amer. Math. Soc. 20 (2007), 799-827.

7. — Quantum dynamics via complex analysis methods: general upper bounds without time-averaging and tight lower bounds for the strongly coupled fibonacci hamiltonian, J. Funct. Anal 255 (2008), 2872-2887.

8. _ A general description of quantum dynamical spreading over an orthonormal basis and applications to Schrödinger operators, Discrete Contin. Dyn. Syst. A 28 (2010), 1381-1412.

9. N. Dunford and J. Schwartz, Linear Operators. Part I. General Theory, Wiley, 1988.

10. D.H. Dunlap, Wu H.-L., and P.W. Phillips, Absence of localization in random-dimer model, Phys. Rev. Lett. 65 (1990), 88-91.

11. F. Germinet, A. Kiselev, and S. Tcheremchantsev, Transfer matrices and transport for Schrödinger operators, Ann. Henri Poincaré 54 (2004), 2872-2887.

12. E. Hamza, R. Sims, and G. Stolz, Dynamical localization in disordered quantum spin systems, Comm. Math. Phys. 315 (2012), 215-239.

13. M.B. Hastings, Locality in quantum and Markov dynamics on lattices and networks, Phys. Rev. Lett. 93 (2004), 140402.

14. S. Jitomirskaya and H. Schulz-Baldes, Upper bounds on wavepacket spreading for random Jacobi matrices, Comm. Math. Phys. 273 (2007), 601-618.

15. S. Jitomirskaya, H. Schulz-Baldes, and G. Stolz, Delocalization in random polymer models, Comm. Math. Phys. 233 (2003), 27-48.

16. R. Killip, A. Kiselev, and Y. Last, Dynamical upper bounds on wavepacket spreading, Amer. J. Math. 125 (2003), 1165-1198.

17. H. Krüger, Positive Lyapunov Exponent for Ergodic Schrödinger Operators, Phd thesis, Rice University, 2010.

18. E. Lieb, T. Schultz, and D. Mattis, Two soluble models of an antiferromagnetic chain, Ann. Phys. 16 (1961), 407-466.

19. E. H. Lieb and D. W. Robinson, The finite group velocity of quantum spin systems, Comm. Math. Phys. 28 (1972), 251-257.

20. L. Marin, Dynamical bounds for Sturmian Schrödinger operators, Rev. Math. Phys. 22 (2010), 859-879.

21. B. Nachtergaele and R. Sims, Lieb-Robinson bounds and the exponential clustering theorem, Comm. Math. Phys. 265 (2006), 119-130.

Mathematics Dept. MS-136, Rice University, Houston, TX 77005

E-mail address: damanik@rice.edu

Mathematics Dept. MC 253-37, California Institute of Technology, Pasadena, CA 91125

E-mail address: mlemm@caltech.edu

Mathematics Dept. MS-136, Rice University, Houston, TX 77005

E-mail address: milivoje.lukic@rice.edu

Mathematics Dept. MS-136, Rice University, Houston, TX 77005

E-mail address: yessen@rice.edu 